\section{Neuropsychiatric manifestations in a child with agenesis of the corpus callosum}

Sir,

The corpus callosum (CC) is the largest commissure of the brain that allows communication between the two hemispheres and is the fastest growing nerve tract in brain evolution. It connects to the contralateral hemisphere, integrating motor, sensory, and cognitive performances of the brain. Agenesis of corpus callosum (ACC) is a congenital defect of the brain in which the 200 million axons of the CC are either completely or partially absent. It is mostly a congenital anomaly that occurs either isolated or in combination with other central nervous system or systemic malformations (e.g., dilated ventricles, the absence of the cingulate gyrus, microgyria, etc.). Once thought to be a rare condition, ${ }^{[1]} \mathrm{ACC}$ has been turning up with increasing frequency ever since the widespread clinical use of magnetic resonance imaging (MRI). The effects of the disorder range from subtle to severe, depending on associated 
brain abnormalities. Children and adolescents with isolated ACC (without other associated brain anomalies) can exhibit intellectual disability, seizures, feeding problems, developmental delay, cognitive problems, learning difficulty, poor motor coordination ${ }^{[2,3]}$ etc., We present a case of a 6-year-old child who presented with various neuropsychiatric manifestations in whom complete ACC was detected.

Master A, 6-year-old child with uneventful birth history without past and family history of neuropsychiatric illness presented with complaints of irritability, hyperactivity, poor impulse control, poor scholastic performance, temper outbursts, crying spells, sleep difficulties from childhood. He also has features suggestive of generalized tonic-clonic seizure episodes from the age of 9 months. These seizure episodes occur at a frequency of 6-10 episodes per year. He has not received any treatment for his above-mentioned problems. His developmental history revealed delayed mile stones. On evaluation, no repetitive or restrictive behavior was observed, and he has no significant language problem. Psychological testing revealed a full-scale intelligence quotient of 40 . A pediatric neurological examination was normal. MRI was done and showed complete callosal agenesis. Thyroid function studies, urine metabolic, amino acid, and organic acid screens were normal. Electroencephalography revealed generalized polyspikes and slow wave discharge at 3-5 Hz, normal background cerebral activity prompting the diagnosis of generalized tonic-clonic epilepsy. He was prescribed $600 \mathrm{mg}$ of sodium valproate in divided doses along with $1 \mathrm{mg}$ of risperidone in divided doses.

The cognitive, behavioral and neurological consequences of ACC are wide-ranging. Our patient has features of intellectual disability, behavioral symptoms along with seizure disorder. His symptoms did not qualify the diagnosis of autism or attention deficit hyperactivity disorder (ADHD) or any other specific psychiatric diagnosis except mentioned above. Recently, ACC has been linked to various neuropsychiatric disorders including
ADHD, schizophrenia, cognitive deficits, obsessivecompulsive disorder, dementia, bipolar disorder ${ }^{[4,5]}$ etc., So neuropsychological and behavioral characterization of ACC may help to clarify distinctions between it and various behavioral disorders (e.g., autism, Tourette's syndrome and ADHD). Research on stem cells may provide in future a more permanent rehabilitation of structural disorders of CC. In future, more studies are required to increase the awareness among the physicians about the occurrence of ACC in behaviorally disturbed children.

Satyakam Mohapatra, Udit Kumar Panda, Alok Jyoti Sahoo, Snehanshu Dey, Neelmadhav Rath

Department of Psychiatry, Mental Health Institute, S.C.B Medical College, Cuttack, Odisha, India

Address for correspondence:
Dr. Satyakam Mohapatra,
Mental Health Institute,
S.C.B Medical College,
Cuttack - 753 007, Odisha, India.
E mail: satyakgmu@gmail.com

\section{References}

1. Chiappedi M, Bejor M. Corpus callosum agenesis and rehabilitative treatment. Ital J Pediatr 2010;36:64.

2. Doherty D, Tu S, Schilmoeller K, Schilmoeller G. Health-related issues in individuals with agenesis of the corpus callosum. Child Care Health Dev 2006;32:333-42.

3. Badaruddin DH, Andrews GL, Bölte S, Schilmoeller KJ, Schilmoeller G, Paul LK, et al. Social and behavioral problems of children with agenesis of the corpus callosum. Child Psychiatry Hum Dev 2007;38:287-302.

4. Lewis SW, Reveley MA, David AS, Ron MA. Agenesis of the corpus callosum and schizophrenia: A case report. Psychol Med 1988;18:341-7.

5. David AS, Wacharasindhu A, Lishman WA. Severe psychiatric disturbance and abnormalities of the corpus callosum: Review and case series. J Neurol Neurosurg Psychiatry 1993;56:85-93.

\begin{tabular}{|l|l|}
\hline \multicolumn{2}{|c|}{ Access this article online } \\
\hline Quick Response Code: & Website: \\
\hline & www.ruralneuropractice.com \\
\cline { 2 - 2 } & \\
\hline & \\
\hline
\end{tabular}

